University of Nebraska - Lincoln

DigitalCommons@University of Nebraska - Lincoln

Faculty Publications, Department of Physics and Astronomy

Research Papers in Physics and Astronomy

2009

\title{
Magnetism of Chromia from First-Principles Calculations
}

Siqi Shi

University of Nebraska - Lincoln

Aleksander L. Wysocki

University of Nebraska-Lincoln, awysocki@iastate.edu

Kirill D. Belashchenko

University of Nebraska-Lincoln, belashchenko@unl.edu

Follow this and additional works at: https://digitalcommons.unl.edu/physicsfacpub

Part of the Physics Commons

Shi, Siqi; Wysocki, Aleksander L.; and Belashchenko, Kirill D., "Magnetism of Chromia from First-Principles Calculations" (2009). Faculty Publications, Department of Physics and Astronomy. 75.

https://digitalcommons.unl.edu/physicsfacpub/75

This Article is brought to you for free and open access by the Research Papers in Physics and Astronomy at DigitalCommons@University of Nebraska - Lincoln. It has been accepted for inclusion in Faculty Publications, Department of Physics and Astronomy by an authorized administrator of DigitalCommons@University of Nebraska Lincoln. 


\title{
Magnetism of chromia from first-principles calculations
}

\author{
Siqi Shi, ${ }^{1,2, *}$ A. L. Wysocki, ${ }^{1}$ and K. D. Belashchenko ${ }^{1}$ \\ ${ }^{1}$ Department of Physics and Astronomy and Nebraska Center for Materials and Nanoscience, University of Nebraska-Lincoln, \\ Lincoln, Nebraska 68588, USA \\ ${ }^{2}$ Department of Physics, Center for Optoelectronics Materials and Devices, Zhejiang Sci-Tech University, Xiasha College Park, \\ Hangzhou 310018, China
}

(Received 21 October 2008; published 5 March 2009)

\begin{abstract}
The electronic structure and magnetism of chromia (corundum-type $\mathrm{Cr}_{2} \mathrm{O}_{3}$ ) are studied using full-potential first-principles calculations. The electronic correlations are included within the LSDA $+U$ method. The energies of different magnetic configurations are very well fitted by the Heisenberg Hamiltonian with strong exchange interaction with two nearest neighbors and additional weak interaction up to the fifth neighbor shell. These energies are insensitive to the position of the oxygen states, indicating that magnetism in $\mathrm{Cr}_{2} \mathrm{O}_{3}$ is dominated by direct exchange. The Néel temperature is calculated using the pair-cluster approximation for localized quantum spins of magnitude $3 / 2$. Very good agreement with experiment is found for all properties including the equilibrium volume, spectral density, local magnetic moment, band gap, and the Néel temperature for the values of $U$ and $J$ that are close to those obtained within the constrained occupation method. The band gap is of the Mott-Hubbard type.
\end{abstract}

DOI: $10.1103 /$ PhysRevB.79.104404

PACS number(s): 75.50.Ee, 75.30.Et, 77.80.Bh

\section{INTRODUCTION}

Corundum-type $\mathrm{Cr}_{2} \mathrm{O}_{3}$ is one of the antiferromagnetic transition-metal oxides which present a challenge for electronic band theory due to the correlated character of the partially filled spin-polarized $3 d$ shell. It also has numerous applications in electronic devices, fuel cell electrodes, gas sensors, heterogeneous catalysis, and thermal barrier coatings. Surface properties of $\mathrm{Cr}_{2} \mathrm{O}_{3}$ are of particular interest. The nominally polar but compensated (0001) surface exhibits structural phase transitions which are poorly understood, ${ }^{1,2}$ as well as an uncompensated surface moment ${ }^{3}$ which may be useful in spintronic applications. It is therefore very desirable to establish whether electronic correlations can be reliably included in first-principles calculations in a way that would accurately predict structural, electronic, and magnetic properties.

As expected for a transition-metal oxide, conventional density-functional theory (DFT) studies of bulk $\mathrm{Cr}_{2} \mathrm{O}_{3}$ (Refs. 4-6) have shown that local spin-density approximation (LSDA) or the generalized-gradient approximation (GGA) for the exchange-correlation potential are unable to reproduce the electronic and magnetic properties of bulk $\mathrm{Cr}_{2} \mathrm{O}_{3}$. Rohrbach et al. ${ }^{7}$ performed a GGA $+U$ calculation for $\mathrm{Cr}_{2} \mathrm{O}_{3}$ using the simplified (spherically averaged) $U-J$ correction $^{8}$ and obtained more reasonable results for the band structure. However, this approach is inaccurate for structural and magnetic properties. First, as is typical for transition-metal compounds, both GGA and the LSDA $+U$ corrections reduce the LSDA overbinding problem in $\mathrm{Cr}_{2} \mathrm{O}_{3}$ and increase the equilibrium volume which becomes close to the experimental value. However, the use of both GGA and Hubbard $U$ in the $\mathrm{GGA}+U \operatorname{method}^{7}$ results in an overcorrection, so that the equilibrium volume becomes $7 \%$ too large. Second, the magnetic energies found by Rohrbach et al. ${ }^{7}$ within this method are incompatible with the experimental Néel temperature of about $308 \mathrm{~K}$; they are too small roughly by a factor of 5 .
Mosey et al. ${ }^{9}$ obtained better overall agreement with experiment for structural and spectral properties using the spherically averaged LSDA $+U \operatorname{method}^{8}$ with $U-J=3.2 \mathrm{eV}$, but the magnetic properties were not considered.

In this paper we analyze the magnetic properties of $\mathrm{Cr}_{2} \mathrm{O}_{3}$ using the LSDA $+U$ method. Computational techniques and the choice of the $U$ and $J$ parameters are explained in Sec. II. The results are presented and discussed in Sec. III. In particular, we calculate the exchange parameters, show that the magnetic interaction is dominated by direct exchange between $\mathrm{Cr}$ states, and find the Néel temperature using the pair-cluster approximation for the quantum spin-3/2 Heisenberg model. Overall, the LSDA $+U$ method provides very good agreement with experiment for the structural and spectral properties and the Néel temperature. The results are briefly summarized in Sec. IV and the Appendix gives the technical details of the pair-cluster approximation.

\section{COMPUTATIONAL DETAILS}

The first-principles calculations were carried out using the projected augmented wave method ${ }^{10,11}$ implemented within the Vienna $a b$ initio simulation package (VASP). ${ }^{12,13}$ The valence basis included $3 d$ and $4 s$ states on $\mathrm{Cr}$ and $2 s$ and $2 p$ states on $\mathrm{O}$. We used the rhombohedral primitive cell for the corundum structure in all calculations except those involving complicated magnetic configurations (Sec. III A), where the hexagonal unit cell was adopted (see Fig. 1). The Monkhorst-Pack scheme ${ }^{14}$ based on the $4 \times 4 \times 4 k$-point grid was employed for the Brillouin-zone integrals, which were calculated using the tetrahedron method with Blöchl corrections. ${ }^{15}$ The cutoff energies for the plane-wave and augmentation charge were 520 and $676 \mathrm{eV}$, respectively. These parameters ensured the total energy convergence to 2 meV/atom. Densities of states (DOSs) were calculated using the $8 \times 8 \times 8 k$-point grid. 


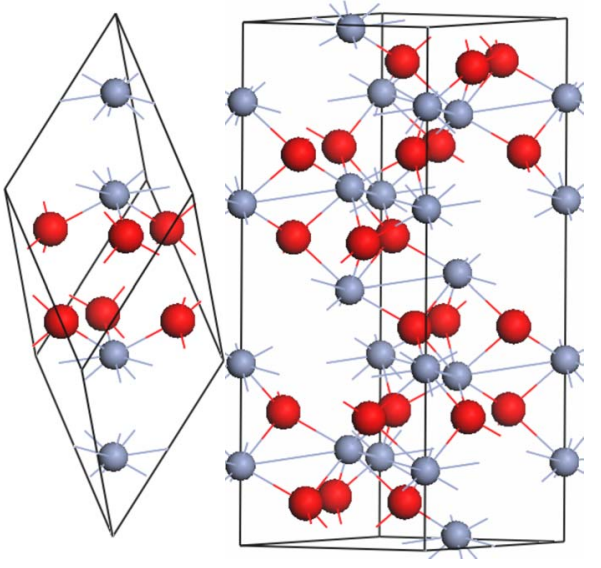

FIG. 1. (Color online) Rhombohedral primitive cell of $\mathrm{Cr}_{2} \mathrm{O}_{3}$ (left) together with the hexagonal unit cell (right). Smaller blue (lighter) spheres represent $\mathrm{Cr}$ atoms; larger red (darker) spheres display the $\mathrm{O}$ atoms.

We employ the LSDA $+U$ method in its full spherically symmetric form. ${ }^{16}$ This extension is important for $\mathrm{Cr}_{2} \mathrm{O}_{3}$ where correct representation of crystal field and exchange splittings within the partially filled $3 d$ shell is critical. Surface energetics are even more sensitive to the correct treatment of these unfilled shells; in fact, we found that the errors introduced by the spherically averaged LSDA $+U$ ansatz are intolerable for the $\mathrm{Cr}_{2} \mathrm{O}_{3}(0001)$ surface. ${ }^{17}$ The doublecounting term is taken in the fully localized limit. ${ }^{16,18}$ The underlying LSDA functional of Perdew and Zunger ${ }^{19}$ is used.

Reasonable values of $U$ and $J$ can usually be obtained within DFT using the constrained occupation method. ${ }^{20} \mathrm{We}$ calculated $U$ and $J$ using the full-potential linear augmented plane-wave (FLAPW) method implemented in the FLEUR package. ${ }^{21}$ In these calculations the GGA approximation was used. We took the rhombohedral primitive cell of $\mathrm{Cr}_{2} \mathrm{O}_{3}$ (see Fig. 1) containing four $\mathrm{Cr}$ atoms and set all the structural parameters to their experimental values. ${ }^{22}$ The $3 d$ electrons on one or two $\mathrm{Cr}$ sites in this cell were treated as "open core" shells, i.e., an integer occupation of these orbitals (for each spin projection) was enforced, and their hybridization with all other electrons was turned off. The $U$ and $J$ parameters were then found by comparing the LSDA total energies for different charge and spin occupations of the $3 d$ orbital(s) to their Hartree-Fock expressions (the latter are equal to the "double-counting" terms in LSDA $+U)$.

The constrained occupation method is somewhat ambiguous because the $U$ parameter depends on the charge state of the ion. ${ }^{23}$ (On the contrary, the $J$ parameter is usually very well defined.) Although the formal charge state of the chromium ion is $\mathrm{Cr}^{3+}$, we find $U$ and $J$ with respect to the $3 d^{4}$ state. The reason is that the $3 d$ wave functions and the screening properties of the valence electrons depend primarily on the charge density in the crystal, which is typically very close to the superposition of atomic charge densities. Indeed, the formal occupancy of the $\mathrm{Cr} 3 d$ orbital within the 2.5 a.u. muffin-tin sphere is about 4.2 in FLAPW. The exchange parameter $J=0.58 \mathrm{eV}$ was found by treating $3 d$ electrons on one $\mathrm{Cr}$ atom as open core and considering the energy differences between the $3 d_{\uparrow}^{2} 3 d_{\downarrow}^{2}, 3 d_{\uparrow}^{3} 3 d_{\downarrow}^{1}$, and $3 d_{\uparrow}^{4} 3 d_{\downarrow}^{0}$

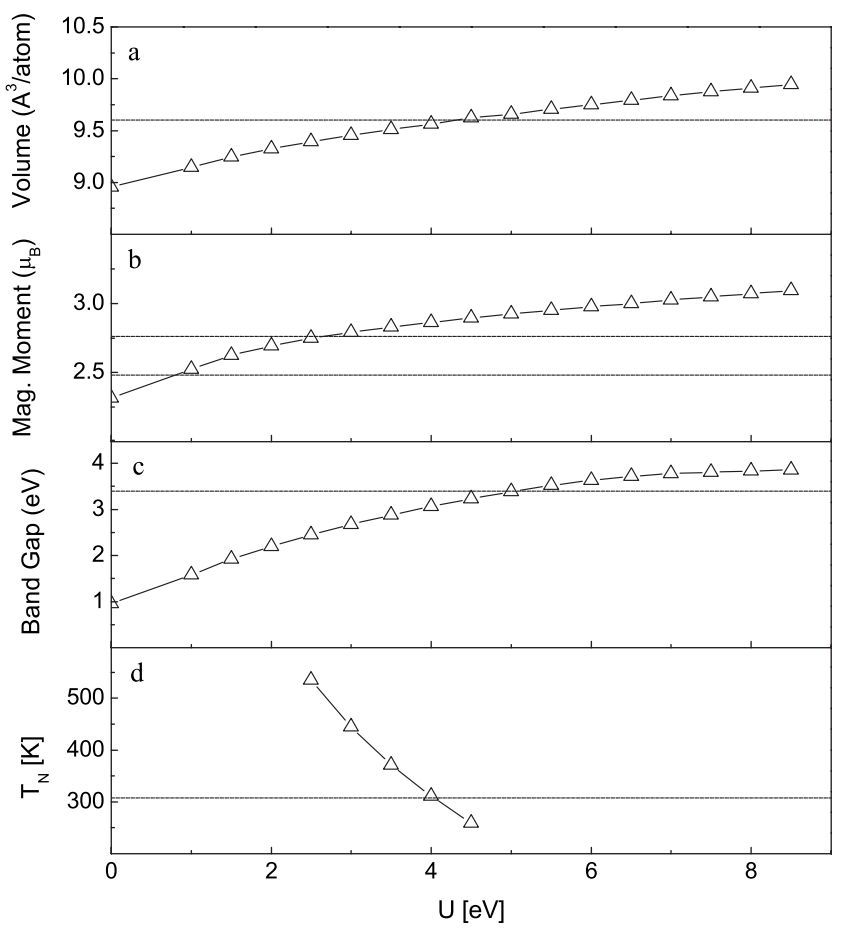

FIG. 2. (a) Atomic volume, (b) magnetic moment, (c) band gap, and (d) Néel temperature as functions of the Coulomb $U$ parameter for the antiferromagnetic +-+- state. The value of $J$ is fixed at 0.58 $\mathrm{eV}$. Horizontal lines denote experimental values; those in (b) are from both Refs. 25 and 26.

configurations. The Hubbard parameter $U=3.3 \mathrm{eV}$ was found by treating the $3 d$ shells on two $\mathrm{Cr}$ sites as open cores and considering the energy difference between the $3 d_{A}^{4} 3 d_{B}^{4}$ and $3 d_{A}^{5} 3 d_{B}^{3}$ configurations, where $A$ and $B$ refer to the two different sites; ${ }^{24}$ the contribution of $J$ to the energy differences was subtracted. Since the total number of electrons in the cell is the same for both configurations, there is no need to include the Fermi-level correction.

Using direct calculations of Coulomb and exchange integrals averaged over the filled molecular orbitals in the unrestricted Hartree-Fock method, Mosey et al. ${ }^{9}$ obtained the values of $U=3.3 \mathrm{eV}$ and $J=0.1 \mathrm{eV}$ for $\mathrm{Cr}_{2} \mathrm{O}_{3}$. Their value of $U-J$ is thus very close to ours, but the $J$ value is much smaller. In the following calculations we fixed $J$ at its calculated value of $0.58 \mathrm{eV}$ and varied $U$ with the expectation that the optimal results should be found for $U \sim 3-4 \mathrm{eV}$.

\section{RESULTS AND DISCUSSION}

The dependence of the equilibrium volume, magnetic moment, and band gap on $U$ for the ground antiferromagnetic state (denoted as +-+- according to the ordering of $\mathrm{Cr}$ spins along the $z$ axis of the rhombohedral cell) is shown in Fig. 2 . The electronic structure was calculated for relaxed structural parameters. We see that the volume and the band gap agree quite well with experimental data at $U=4 \mathrm{eV}$. Other structural parameters also agree with experiment. The calculated angle between the rhombohedral unit vectors is $55.11^{\circ} \mathrm{com}-$ pared to the measured ${ }^{22}$ angle of $55.13^{\circ}$. The shortest dis- 


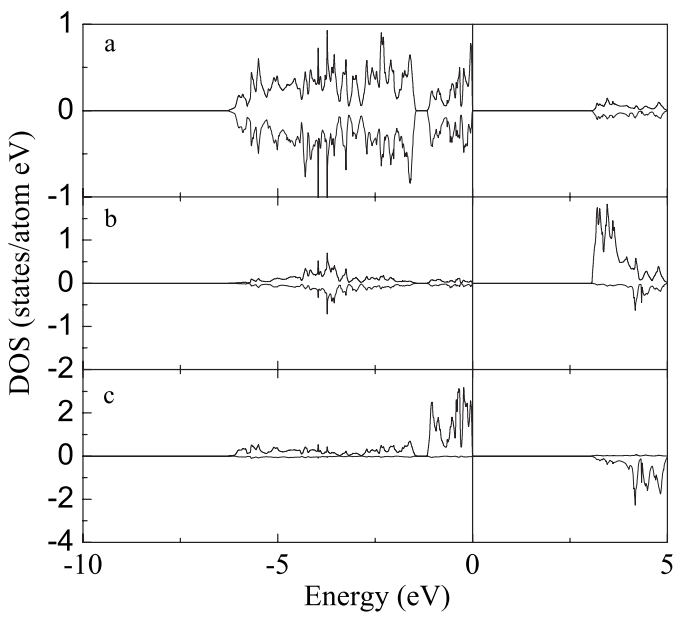

FIG. 3. Orbital-projected DOS for the antiferromagnetic +-+ground state of bulk $\mathrm{Cr}_{2} \mathrm{O}_{3}$, calculated within the LSDA $+U$ with $U=4.0 \mathrm{eV}$ and $J=0.58 \mathrm{eV}$. (a) $\mathrm{O} 2 p$, (b) $\mathrm{Cr} e_{g}$, and (c) $\mathrm{Cr} t_{2 g}$. Majority- and minority-spin DOSs are plotted with different signs.

tance between $\mathrm{Cr}$ atoms along the [111] axis is $2.646 \AA$ vs the measured $2.650 \AA$.

The magnetic moment at $U=4.0 \mathrm{eV}$ is $2.86 \mu_{B}$, i.e., it is somewhat reduced compared to the "ideal" ionic value of $3 \mu_{B}$ corresponding to a fully localized spin $3 / 2 .{ }^{27}$ Experimentally, the most recent neutron polarimetry measurement gives the sublattice magnetization of $2.48 \mu_{B},{ }^{25}$ which is notably lower compared to an older estimate of $2.76 \mu_{B} .{ }^{26}$ The magnetic moment is smaller than $3 \mu_{B}$ due to two effects: (1) hybridization with oxygen, which is included in our calculation, and (2) the quantum "zero-point spin deviation," which is absent in DFT. The zero-point deviation in $\mathrm{Cr}_{2} \mathrm{O}_{3}$ was estimated $^{28}$ to be about $8 \%$, which amounts to $0.24 \mu_{B}$. Thus, keeping in mind the uncertainties related to the definition, measurement, and calculation of the magnetic moment, its calculated value at $U \approx 4.0 \mathrm{eV}$ is completely reasonable. Of course, the poorly defined reduction from $3 \mu_{B}$ cannot be used as an indicator of the quality of agreement with experiment. We also note that the local magnetic moment depends very weakly on the magnetic configuration of $\mathrm{Cr}_{2} \mathrm{O}_{3}$. In the ferromagnetic state the local moment within the muffin-tin sphere is $2.94 \mu_{B}$, while the magnetization is exactly equal to $3 \mu_{B}$ per $\mathrm{Cr}$ site, as expected. The orbital moment in the calculation with spin-orbit coupling is small (about $0.04 \mu_{B}$ ) and antiparallel to the spin moment, in agreement with the experimental ${ }^{29} \mathrm{~g}$ factor of 1.97 and with the general rule for atomic shells that are less than half filled.

The band gap of $3.07 \mathrm{eV}$ is somewhat smaller than the experimental value of $3.4 \mathrm{eV}$, but greater than that found in Ref. 7 using GGA $+U$ with $U-J=4 \mathrm{eV}$. Underestimation of the addition energy is a common feature of the LSDA $+U$ method, which is well known, for example, for Gd and other $4 f$ elements. Further, the density of states shown in Fig. 3 is in excellent agreement with x-ray photoemission data. ${ }^{30,31}$ Namely, the sharp and narrow peak at low binding energies separated by a (pseudo)gap from the rest of the valence band is very well reproduced. Figure 3 also shows the partial DOS decomposition into $\mathrm{O}$ and $\mathrm{Cr}$ contributions from $t_{2 g}$ and $e_{g}$ states. (The $t_{2 g}$ and $e_{g}$ subbands are well defined because the
TABLE I. The exchange parameters $J_{n}$ fitted using the total energies of 12 magnetic configurations calculated for different values of $U$ with $J$ fixed at $0.58 \mathrm{eV}$. Each pair of sites contributes $J_{i j} \mathbf{e}_{i} \mathbf{e}_{j}$ to the total energy, where $\mathbf{e}_{i}$ is the unit vector parallel to the direction of the local moment at site $i$. The last column $\Delta$ shows the mean-square misfit in the fitting of the total energy. $J_{n}$ and $\Delta$ are given in $\mathrm{meV}$.

\begin{tabular}{ccccccc}
\hline \hline$U$ & $J_{1}$ & $J_{2}$ & $J_{3}$ & $J_{4}$ & $J_{5}$ & $\Delta$ \\
\hline 2.5 & 30.9 & 21.9 & -0.60 & -1.83 & 4.92 & 0.91 \\
3.0 & 23.9 & 17.3 & -1.26 & -2.36 & 3.72 & 0.62 \\
3.5 & 18.6 & 13.8 & -1.74 & -2.72 & 2.84 & 0.43 \\
4.0 & 14.6 & 11.1 & -2.11 & -2.96 & 2.16 & 0.30 \\
4.5 & 11.1 & 9.04 & -2.41 & -3.11 & 1.64 & 0.20 \\
\hline \hline
\end{tabular}

ligand field of the $\mathrm{Cr}$ site is approximately octahedral. The Cartesian axes are selected accordingly.) As seen from Fig. 3, the peak at low binding energies corresponds to the filled $\mathrm{Cr} t_{2 g}$ spin subband with some admixture of oxygen $p$ orbitals. At least some of this admixture is fictitious because the $\mathrm{Cr} d$ orbitals extend into oxygen's projection spheres. (The same ambiguity is involved in the definition of the $\mathrm{Cr}$ magnetic moment.) Since nonmagnetic $\mathrm{Cr}_{2} \mathrm{O}_{3}$ is metallic with the Fermi level lying inside the $t_{2 g}$ subband which is separated by a gap from the oxygen $p$ band, the insulating gap forms by the Mott-Hubbard mechanism.

Thus, the structural and spectral properties of $\mathrm{Cr}_{2} \mathrm{O}_{3}$ are well reproduced by the spherically symmetric LSDA $+U$ method with $U \approx 4.0 \mathrm{eV}$ and $J=0.58 \mathrm{eV}$. This value of $U$ is reasonably close to that given by the constrained occupation method.

\section{A. Exchange parameters}

We now focus on the magnetic energetics which provides another stringent test of the validity of the LSDA $+U$ method for $\mathrm{Cr}_{2} \mathrm{O}_{3}$. We calculated the total energies of 12 different magnetic configurations including the ferromagnetic state, three simple antiferromagnetic orderings $(+-+-,++--$, and $+--+)$, and eight additional arbitrarily chosen spin configurations in the hexagonal unit cell (Fig. 1) which contains six formula units. The ground antiferromagnetic state has +-+- ordering in agreement with experiment; its relaxed structure was fixed for other magnetic orderings. The calculated total energies are well fitted by the conventional Heisenberg Hamiltonian with exchange interaction in five coordination spheres. The exchange parameters fitted by the least-squares method for different values of $U$ are listed in Table I. The first, second, and fifth nearest-neighbor interactions are antiferromagnetic, whereas the third and fourth ones are ferromagnetic. All exchange parameters except the third one reinforce the ground state. Also, we see that the first and second exchange parameters are significantly larger than those for more distant neighbors; this behavior is natural for an insulator. $\mathrm{In}_{\mathrm{Cr}_{2} \mathrm{O}_{3}}$ the parameter $J_{1}$ corresponds to the short bond along the $z$ axis and $J_{2}$ to the nearest-neighbor bond within the buckled $\mathrm{Cr}$ layer. Each $\mathrm{Cr}$ atom has one $J_{1}$ neighbor and three $J_{2}$ neighbors. As the value of $U$ is in- 
creased, all antiferromagnetic exchange parameters systematically decrease, while the ferromagnetic parameters systematically increase.

Let us compare our results to those derived from the spinwave dispersion measured by inelastic neutron scattering. ${ }^{28}$ The exchange parameters fitted to those dispersion curves are also dominated by antiferromagnetic $J_{1}$ and $J_{2}$. However, more distant neighbors in that fitting ${ }^{28}$ are much less important. For example, $J_{5} / J_{1} \approx 1 / 40$ in Ref. 28 vs $1 / 7$ found here. Also, there is a notable disagreement in the $J_{2} / J_{1}$ ratio: Ref. 28 found $J_{2} / J_{1} \approx 0.45$ vs 0.76 for $U=4 \mathrm{eV}$ in Table I.

We have verified that the anisotropy $J_{2} / J_{1}$ is almost unchanged if only the lowest-lying spin configurations are included in the fitting. We also considered the possibility that the exchange parameters may be affected by lattice distortion below the Néel temperature due to magnetostructural coupling. In order to study this possibility, we need to know how the exchange parameters depend on the structural parameters and how the latter change between room temperature and liquid nitrogen temperature where the spin-wave spectrum was measured. We found that the values of $J_{1}$ and $J_{2}$ are very sensitive to the lattice parameters; a $1 \%$ increase or decrease in the first- or second-neighbor bond length leads to a $25 \%-$ $50 \%$ decrease or increase of the corresponding exchange parameter (this was roughly established by varying the Bravais lattice parameters of the rhombohedral cell and fitting the four simple magnetic orderings to the Heisenberg model). Thus, the $J_{2} / J_{1}$ ratio is very sensitive to the $c / a$ ratio (or any lattice distortion that changes the ratio of the first and second bond lengths $R_{2} / R_{1}$ ).

To our knowledge, no experimental data are available on the thermal contraction of $\mathrm{Cr}_{2} \mathrm{O}_{3}$ below room temperature. In order to assess the degree of magnetostructural coupling, we have fully relaxed the structure for all the 12 magnetic configurations that were included in the fitting of exchange parameters. The magnetostructural coupling is, in general, quite appreciable; the $R_{2} / R_{1}$ ratio varies between the minimum of 1.072 in the +--+ configuration (where the nearest-neighbor pairs are all parallel and next-nearest antiparallel) and the maximum of 1.103 in the ++-- configuration (where the situation is reversed). The overall trend, as expected in general for antiferromagnetic coupling, is for each of these bonds to shorten when the corresponding spins are antiparallel and lengthen when they are parallel. Although this effect seems to be rather large, the actual +-+- state has both the first- and second-neighbor pairs antiparallel, so that both should lengthen somewhat in the paramagnetic state. Among our 12 configurations, five have the same ratio $N_{\mathrm{P}}^{(i)} / N_{\mathrm{AP}}^{(i)}$ of the number of parallel and antiparallel pairs in the $i$ th coordination sphere for $i=1$ and $i=2$, i.e., these five configurations approximately represent the change of structure as a function of the antiferromagnetic order parameter. The $R_{2} / R_{1}$ ratio for these five structures varies between 1.088 in the +-+- state and 1.084 in the ferromagnetic state. The three intermediate states, all of which have twice as many parallel pairs than antiparallel ones, all have $R_{2} / R_{1}=1.087$. The bond lengths themselves change by about $0.7 \%$ between the +-+- state and these three intermediate states. This variation is obviously too small to explain the disagreement with experiment in the $J_{2} / J_{1}$ ratio.
Thus, in spite of the overall agreement with experiment for most properties, a discrepancy in the $J_{2} / J_{1}$ ratio remains. It is possible that our Heisenberg model fitting is not fully applicable to small deviations from the ground state, for which the linear-response technique should be a better fit. Further, spin waves are more sensitive to distant couplings compared to the energy fits or thermodynamic properties, which makes the fits from spin-wave data and from the overall energetics statistically inequivalent. Finally, many-body effects beyond LSDA $+U$ may play a role in renormalizing the exchange parameters. We also note that impurities and thermal spin disorder should be more effective in destroying distant couplings compared to nearest-neighbor ones; this may explain the larger role of couplings beyond $J_{2}$ in our fitting compared to experimental spin-wave results.

\section{B. Mechanism of magnetic interaction}

The mechanisms of exchange interaction in $\mathrm{Cr}_{2} \mathrm{O}_{3}$ are not well understood. Complicated crystal structure with the presence of many electronic orbitals of different symmetries and many $\mathrm{Cr}-\mathrm{O}-\mathrm{Cr}$ links at different angles greatly complicates the empirical analysis. Both superexchange and direct exchange interactions have been invoked to explain the magnetic structure of $\mathrm{Cr}_{2} \mathrm{O}_{3} \cdot{ }^{32-35}$ Direct exchange interaction may be interpreted in terms of hopping of electrons from one $\mathrm{Cr}$ ion to another across the insulating gap; the energy denominator involved in this process is the Mott-Hubbard splitting. The hopping can only be effective between the orbitals that are able to hybridize. From the DOS decomposition shown in Fig. 3 it is clear that the $t_{2 g}$ orbitals very weakly hybridize with $e_{g}$ orbitals on the neighboring $\mathrm{Cr}$ atoms. Therefore, the contribution of $e_{g}$ orbitals to direct exchange can be neglected. The $t_{2 g}$ subband is split off by crystal field and exactly half filled, therefore direct exchange should be antiferromagnetic. The superexchange involves hopping between $\mathrm{Cr}$ and $\mathrm{O}$ ions; the energy denominator involves the gap between the oxygen $p$ states and the unoccupied $\mathrm{Cr}$ states.

In order to reveal the mechanism responsible for exchange interaction in $\mathrm{Cr}_{2} \mathrm{O}_{3}$, we use the following procedure. A fictitious external potential $V$ is coupled to the oxygen $p$ orbitals, which adds the term $E_{V}=V \operatorname{Tr} n_{m m^{\prime}}^{\sigma}$ to the total energy, where the trace is taken over orbital and spin indices, and $n_{m m^{\prime}}^{\sigma}$ is the density matrix of the oxygen $p$ states defined inside the muffin-tin sphere of 1.2 a.u. This density matrix is calculated using the standard LSDA $+U$ machinery. When $V$ is set to a negative value, the filled oxygen $p$ states are pushed down to lower energies, which suppresses superexchange, but not direct exchange. Weak hybridization between the filled $t_{2 g}$ states and the oxygen $p$ states pushes them apart at $V=0$. When the $p$ states are pushed down by increasing the magnitude of $V$, this repulsion is reduced and the $t_{2 g}$ states also move down, thereby increasing the band gap. Since direct exchange is sensitive to the band gap, for better comparison we compensate this increased band gap by reducing $U$ on $\mathrm{Cr}$. This is done in such a way that the distance between the center of mass of the filled $t_{2 g}$ band and the 
TABLE II. Energies of three simple magnetic configurations relative to the ground +-+- state (in $\mathrm{meV}$ per formula unit) as a function of the fictitious external potential $V$ applied to the oxygen $p$ orbitals (in eV). Reduced values of $U$ on $\mathrm{Cr}$ compensate for the increased band gap (see text for details). $\Delta$ is the distance from the oxygen band top to CBM in $\mathrm{eV}$.

\begin{tabular}{cccccc}
\hline \hline$V$ & $U$ & $\Delta$ & ++++ & ++-- & +--+ \\
\hline 0 & 3.5 & 4.5 & 130 & 124 & 66 \\
-12 & 3.5 & 5.8 & 115 & 102 & 59 \\
-12 & 2.5 & 5.6 & 154 & 128 & 74 \\
-24 & 3.5 & 7.9 & 102 & 80 & 51 \\
-24 & 2.15 & 7.7 & 145 & 110 & 71 \\
\hline \hline
\end{tabular}

conduction-band minimum $(\mathrm{CBM})$ remains the same as at $V=0$.

Starting from the state with $U=3.5 \mathrm{eV}$, we added $V$ of -12 and $-24 \mathrm{eV}$ and calculated the energies of four magnetic states in the rhombohedral primitive cell of $\mathrm{Cr}_{2} \mathrm{O}_{3}$. These calculations were performed using the FLAPW method ${ }^{21}$ the results are listed in Table II. (Those for $V=0$ are within $10 \%$ of VASP results.) The self-consistent downward shift of the $p$ states is much smaller than $V n_{m m}$ because $V$ is strongly screened. The distance $\Delta$ from the oxygen $p$ band maximum to CBM is also listed in the table.

The results listed in Table II clearly show that the magnetic energies are insensitive to the position of the oxygen $p$ band. The reduction of magnetic energies produced by adding negative $V$ at constant $U$ is due to the fact that the $t_{2 g}$ band gap increases due to dehybridization from oxygen. Once $U$ is decreased to bring the band gap to its original value, the magnetic energies are essentially unchanged compared to their values at $V=0$; in fact, they even increase somewhat. On the other hand, we have seen above that the magnetic energies are very sensitive to the value of $U$ which is responsible for the band gap. This behavior leads us to a striking conclusion that, contrary to the common belief, superexchange plays no role in magnetism of $\mathrm{Cr}_{2} \mathrm{O}_{3}$. Antiferromagnetism is due exclusively to direct exchange, which, as mentioned above, is antiferromagnetic because the magnetically active $t_{2 g}$ subband is half filled. It is likely that superexchange in $\mathrm{Cr}_{2} \mathrm{O}_{3}$ is highly ineffective because the $\mathrm{Cr}-\mathrm{O}-\mathrm{Cr}$ angles are close to $90^{\circ}$, while the overlap between $\mathrm{O}$ states and $\mathrm{Cr} t_{2 g}$ states is small. On the other hand, the overlaps between $t_{2 g}$ states on neighboring $\mathrm{Cr}$ atoms are quite large; the $t_{2 g}$ bandwidth in the ferromagnetic state at $V=-24 \mathrm{eV}$ is approximately $1.5 \mathrm{eV}$.

\section{Néel temperature}

We now calculate the Néel temperature $T_{N}$. We saw above that the local moments on $\mathrm{Cr}$ atoms are well localized and the energies of spin configurations are well represented by the Heisenberg spin Hamiltonian. We therefore adopt the quantum Heisenberg model for localized spins of magnitude $3 / 2$ with the exchange parameters listed in Table I. Since each spin is strongly coupled only to four neighbors (one with $J_{1}$ and three with $J_{2}$ ), the mean-field approximation can- not be reliably used. However, the antiferromagnetic interaction is not frustrated. The important spin correlations should be generated by the dominant exchange interaction with four nearest neighbors. The network of bonds corresponding to $J_{1}$ and $J_{2}$ is very weakly connected; the shortest closed path on this network is six bonds long. Therefore, it is sufficient to capture the pairwise spin correlations. In this situation, the pair-cluster approximation appears to be an obvious choice. This approach is a special case of the cluster-variation method when the set of clusters includes only pairs of sites. Here we follow the formulation of Ref. 36 which can be directly applied to our case. The details of this technique are described in the Appendix.

The calculated $T_{N}$ is shown in Fig. 2 as a function of $U$. We see that the best agreement with experiment for $T_{N}$ is obtained at the same value of $U \approx 4 \mathrm{eV}$ as for the structural and spectral properties explored above. This overall agreement is a strong indication that the essential details of the electronic structure of $\mathrm{Cr}_{2} \mathrm{O}_{3}$ are very well captured by the LSDA $+U$ approximation. Physically, this success of LSDA $+U$ is explained by the presence of fully filled and empty subbands which are strongly split by crystal and exchange fields; LSDA $+U$ usually reproduces such closed atomiclike subshells very well.

\section{CONCLUSION}

In conclusion, we found that the spherically symmetric LSDA $+U$ method provides a very good description of structural, spectral, and magnetic properties of chromia with $J$ $=0.58 \mathrm{eV}$ found from the constrained occupation method and $U \approx 4 \mathrm{eV}$, which is also close to the calculated value. We found that the magnetic energies are well represented by a Heisenberg model with strong exchange interaction with nearest neighbors both in the buckled layer and along the $z$ axis and much weaker interaction with more distant neighbors. The artificial downward shift of the filled oxygen $p$ states has almost no effect on the magnetic energies, which proves that direct exchange is the dominant mechanism of magnetic interaction.

\section{ACKNOWLEDGMENTS}

This work was supported by NRC/NRI supplement to NSF-MRSEC and by the Nebraska Research Initiative. K.D.B. is a Cottrell Scholar of Research Corporation.

\section{APPENDIX}

Here we describe the application of the pair-cluster approximation to $\mathrm{Cr}_{2} \mathrm{O}_{3}$ following a similar formalism of Ref. 36. The energy of a quantum Heisenberg magnet (per unit cell) can be written as

$$
E=-\frac{1}{2} \sum_{i, j} m_{i} n_{i j} J_{i j}\left\langle\hat{\mathbf{S}}_{i} \cdot \hat{\mathbf{S}}_{j}\right\rangle-\sum_{i} m_{i} B_{i}\left\langle\hat{S}_{i}^{z}\right\rangle,
$$

where the summations are over the inequivalent sites in the unit cell, $\hat{\mathbf{S}}_{i}$ are quantum spin operators, $m_{i}$ is the number of sites of type $i$ in the unit cell, $n_{i j}$ the number of neighbors of 
site $i$ that are of type $j$, and $B_{i}$ the external magnetic field applied to site type $i$. All the thermodynamic properties can be obtained from the free energy which may be calculated by integrating the Gibbs-Helmholtz relation

$$
F=\frac{1}{\beta} \int_{0}^{\beta} E\left(\beta^{\prime}\right) d \beta^{\prime} .
$$

To proceed we need to find the expectation values appearing in Eq. (A1). In the pair-cluster approximation they are calculated by introducing one- and two-site clusters with the following cluster Hamiltonians

$$
\begin{gathered}
\hat{H}_{1}^{i}=-h_{i} \hat{S}_{i}^{z}, \\
\hat{H}_{2}^{i j}=-J_{i j} \hat{\mathbf{S}}_{i} \cdot \hat{\mathbf{S}}_{j}-h_{i}^{(j)} \hat{S}_{i}^{z}-h_{j}^{(i)} \hat{S}_{j}^{z},
\end{gathered}
$$

where $h_{i}=B_{i}+\phi_{i}$ is the one-site "cluster field" and $h_{i}^{(j)}=h_{i}$ $-\phi_{i}^{(j)}$ is the cluster field at site $i$ for the pair cluster $(i, j)$. The one-site and two-site cluster fields are related through $\phi_{i}$ $=\sum_{j} n_{i j} \phi_{i}^{(j)}$. The quantities $\phi_{i}^{(j)}$ are treated as variational parameters and found by minimizing the free energy, i.e., requesting that $\partial F / \partial \phi_{i}^{(j)}=0$. It can be shown that this variational condition ensures that the expectation value $\left\langle\hat{S}_{i}^{z}\right\rangle$ is the same in all clusters containing site $i$. The expectation values $\left\langle\hat{\mathbf{S}}_{i} \cdot \hat{\mathbf{S}}_{j}\right\rangle$ are calculated from the pair cluster $(i, j)$. Performing the integration in Eq. (A2), we find

$$
F=-\frac{1}{2 \beta} \sum_{i j} m_{i} n_{i j} \ln Z_{2}^{i j}+\frac{1}{\beta} \sum_{i} m_{i}\left(n_{i}-1\right) \ln Z_{1}^{i},
$$

where $n_{i}=\sum_{j} n_{i j}$ is the total number of neighbors of site $i$, while $Z_{1}^{i}=\operatorname{Tr} \exp \left(-\beta H_{1}^{i}\right)$ and $Z_{2}^{i j}=\operatorname{Tr} \exp \left(-\beta H_{2}^{i j}\right)$ are the onesite and two-site cluster partition sums. Evaluation of $Z_{1}^{i}$ is trivial; to find $Z_{2}^{i j}$ one first needs to diagonalize $H_{2}^{i j}$.

Our goal here is to find the transition temperature, therefore we may assume that all $h_{i}^{(j)}$ are small. The free energy is developed in these parameters, and then the parameters $\phi_{i}^{(j)}$ are found by requiring that the variation of the free energy $F$ vanishes. (The resulting equations are too cumbersome to be included here.)

For $\mathrm{Cr}_{2} \mathrm{O}_{3}$ we assume the actual magnetic ordering +-+- . All $\mathrm{Cr}$ sites are related by magnetic symmetry, which reduces the number of independent variational parameters. The transition temperature is found by setting $S=3 / 2$ and searching for the pole of the magnetic susceptibility which is found from the one-site cluster

$$
\chi_{i j}=\frac{\partial\left\langle S_{i}^{z}\right\rangle}{\partial B_{j}}=\frac{S(S+1)}{3} \beta \frac{\partial h_{i}}{\partial B_{j}} .
$$

The resulting equation for the transition temperature has two solutions. The greater solution is $T_{N}$, while the lower one is the fictitious "anti-Néel point" below which $\left\langle\hat{S}_{i}^{z}\right\rangle=0$. The existence of an anti-Néel point for antiferromagnets is a well-known shortcoming of the pair-cluster approximation, ${ }^{36,37}$ which fails at low temperatures. However, in our case the anti-Néel point is much smaller than $T_{N}$ which indicates that the pair-cluster approximation has a wide range of validity. Therefore, this method is expected to provide a very good approximation for $T_{N}$.

\footnotetext{
*siqishi@yahoo.com

${ }^{1}$ M. Bender, D. Ehrlich, I. N. Yakovkin, F. Rohr, M. Bäumer, H. Kuhlenbeck, H.-J. Freund, and V. Staemmler, J. Phys.: Condens. Matter 7, 5289 (1995).

${ }^{2}$ Th. Gloege, H. L. Meyerheim, W. Moritz, and D. Wolf, Surf. Sci. 441, L917 (1999).

${ }^{3}$ S. Sahoo and Ch. Binek, Philos. Mag. Lett. 87, 259 (2007).

${ }^{4}$ M. Catti, G. Sandrone, G. Valerio, and R. Dovesi, J. Phys. Chem. Solids 57, 1735 (1996).

${ }^{5}$ A. Y. Dobin, W. Duan, and R. M. Wentzcovitch, Phys. Rev. B 62, 11997 (2000).

${ }^{6}$ K. Wolter, D. Scarano, J. Fritsch, H. Kuhlenbeck, A. Zecchina, and H.-J. Freund, Chem. Phys. Lett. 320, 206 (2000).

${ }^{7}$ A. Rohrbach, J. Hafner, and G. Kresse, Phys. Rev. B 70, 125426 (2004).

${ }^{8}$ S. L. Dudarev, G. A. Botton, S. Y. Savrasov, C. J. Humphreys, and A. P. Sutton, Phys. Rev. B 57, 1505 (1998).

${ }^{9}$ N. J. Mosey, P. Liao, and E. A. Carter, J. Chem. Phys. 129, 014103 (2008).

${ }^{10}$ P. E. Blöchl, Phys. Rev. B 50, 17953 (1994).

${ }^{11}$ G. Kresse and D. Joubert, Phys. Rev. B 59, 1758 (1999).

${ }^{12}$ G. Kresse and J. Hafner, Phys. Rev. B 48, 13115 (1993).

${ }^{13}$ G. Kresse and J. Furthmüller, Phys. Rev. B 54, 11169 (1996).
}

${ }^{14}$ H. Monkhorst and J. D. Pack, Phys. Rev. B 13, 5188 (1976).

${ }^{15}$ P. E. Blöchl, O. Jepsen, and O. K. Andersen, Phys. Rev. B 49, 16223 (1994).

${ }^{16}$ A. I. Liechtenstein, V. I. Anisimov, and J. Zaanen, Phys. Rev. B 52, R5467 (1995).

${ }^{17}$ Siqi Shi and K. D. Belashchenko (unpublished).

${ }^{18}$ A. G. Petukhov, I. I. Mazin, L. Chioncel, and A. I. Lichtenstein, Phys. Rev. B 67, 153106 (2003).

${ }^{19}$ J. P. Perdew and A. Zunger, Phys. Rev. B 23, 5048 (1981).

${ }^{20}$ P. H. Dederichs, S. Blügel, R. Zeller, and H. Akai, Phys. Rev. Lett. 53, 2512 (1984); O. Gunnarsson, O. K. Andersen, O. Jepsen, and J. Zaanen, Phys. Rev. B 39, 1708 (1989); V. I. Anisimov and O. Gunnarsson, Phys. Rev. B 43, 7570 (1991).

${ }^{21}$ S. Blügel and G. Bihlmayer, in Computational Nanoscience: Do It Yourself! edited by J. Grotendorst, S. Blügel, and D. Marx, NIC Series Vol. 31 (John von Neumann Institite for Computing, Jülich, 2006), p. 85; www.flapw.de

${ }^{22}$ L. W. Finger and R. M. Hazen, J. Appl. Phys. 51, 5362 (1980).

${ }^{23}$ I. V. Solovyev and P. H. Dederichs, Phys. Rev. B 49, 6736 (1994).

${ }^{24}$ These two sites are selected so that they are not nearest neighbors along the $z$ axis, which makes the shortest distance between them in the crystal as large as possible. 
${ }^{25}$ P. J. Brown, J. B. Forsyth, E. Lelièvre-Berna, and F. Tasset, J. Phys.: Condens. Matter 14, 1957 (2002).

${ }^{26}$ L. M. Corliss, J. M. Hastings, R. Nathans, and G. Shirane, J. Appl. Phys. 36, 1099 (1965).

${ }^{27}$ Refs. 7 and 9 concluded that there is a large disagreement with experiment in the magnetic moment, but they used an incorrect experimental value of $3.8 \mu_{B}$.

${ }^{28}$ E. J. Samuelsen, M. T. Hutchings, and G. Shirane, Physica (Amsterdam) 48, 13 (1970).

${ }^{29}$ S. Foner, Phys. Rev. 130, 183 (1963).

${ }^{30}$ R. Zimmermann, P. Steiner, and S. Hüfner, J. Electron Spectrosc.
Relat. Phenom. 78, 49 (1996).

${ }^{31}$ T. Uozumi, K. Okada, A. Kotani, R. Zimmermann, P. Steiner, S. Hüfner, Y. Tezuka, and S. Shin, J. Electron Spectrosc. Relat. Phenom. 83, 9 (1997).

${ }^{32}$ Y. Y. Li, Phys. Rev. 102, 1015 (1956).

${ }^{33}$ S. Iida, J. Phys. Soc. Jpn. 11, 1300 (1956).

${ }^{34}$ J. B. Goodenough, Phys. Rev. 117, 1442 (1960).

${ }^{35}$ W. P. Osmond, Proc. Phys. Soc. London 79, 394 (1962).

${ }^{36}$ V. G. Vaks and N. E. Zein, Sov. Phys. JETP 40, 537 (1975).

${ }^{37}$ P. W. Kasteleijn and J. van Kranendonk, Physica (Amsterdam) 22, 367 (1956). 